\title{
The Clinical Enquiry Service; Benign intracranial hypertension
}

\author{
Joan Walsh, BSc, Connie Smith, MB, BS, MFFP, Director, Clinical Effectiveness Unit \\ Clinical Effectiveness Unit, Faculty of Family Planning and Reproductive Health Care, London, UK
}

Considering how uncommon the condition is (3.3 cases per 100000 women aged 15 to 44 years ${ }^{1}$ ) the CEU has received a surprising number of enquiries about contraceptive options for women with a diagnosis of idiopathic benign intracranial hypertension.

Does benign intracranial hypertension contraindicate use of contraceptive steroids?

\section{Dear Doctor}

Regarding: Benign intracranial hypertension (also known as pseudotumor cerebri) and use of combined and progestogen-only contraceptive methods.

Thank you for your enquiry. As you observe, benign intracranial hypertension $(\mathrm{BIH})$ is by no means a universally 'benign' condition, being a recognised cause of (usually) reversible visual loss. Raised intracranial pressure is most commonly diagnosed in women of reproductive age, with obese women being particularly at risk. A diagnosis of BIH is associated with numerous conditions, diseases and drug treatments, including endocrine disorders, infectious agents, vitamin A deficiency or overdosage, iron deficiency anaemia, use of tetracyclines, withdrawal of corticosteroid therapy, and thrombotic partial occlusion of cerebral vasculature. Raised intracranial pressure can compromise cerebral blood flow, and there is a small but consistent body of evidence which suggests that idiopathic intracranial hypertension is associated with the presence of underlying coagulation/ haemostasis disorders, such as antiphospholipidantibodies or antithrombin III deficiency. ${ }^{2-5}$

Raised intracranial pressure can cause serious and sometimes permanent loss of sight, so it would be appropriate to eliminate actual or potential risk factors as far as practicable. Given that the BIH is associated with cerebral thrombotic conditions/predispositions, we recommend that any history of this condition represents an unacceptable health risk for use of the combined pill (i.e. World Health Organisation category 4). ${ }^{6}$

As you will know, progestogens used in contraceptive doses have not been shown to be associated with a significant increase in the risk of venous thrombosis, or of stroke in normotensive women. The data are, however, very limited. They do show that the effect of progestogens on the risk of venous thrombosis and stroke, if any, is small. They do not provide conclusive evidence that contraceptive progestogens have no effect on risk. ${ }^{7}$ There are reports which suggest a possible relationship between use of Norplant ${ }^{\circledR}$ and hospitalisation for $\mathrm{BIH},{ }^{8,9}$ although confounding factors (notably obesity) were evident in some of the reported cases and no causal relationship with use of levonorgestrel has been established.

The physiological mechanisms of $\mathrm{BIH}$ are not well understood. There is no evidence available regarding the effect of progestogen-only contraceptive methods on the symptoms or the clinical course of $\mathrm{BIH}$, and it is not possible to predict whether progestogens might have a clinically significant effect in any or all cases. We therefore recommend that BIH should be regarded as a condition in which the potential risks of using progestogen-only methods generally outweigh the benefits (i.e. World Health Organisation category 3 ). ${ }^{6}$ The acceptability and user-efficacy of non-hormonal methods and the risks of pregnancy would, of course, need to be taken into consideration.
In cases where a treatable or self-limiting cause of BIH had been identified, and where thrombotic association had been excluded, a history of $\mathrm{BIH}$ would not necessarily contraindicate use of contraceptive progestogens. Whether or not the benefits of using a progestogen-only method outweighed a small potential (perhaps non-existent) risk of recurrence would depend on the individual client's history, priorities and concerns. After consideration of all these factors and of all the non-hormonal contraceptive options, a fully informed woman with a history of BIH without thrombotic association might choose to use a progestogenonly method. It would clearly be very important for her to be aware of any signs and symptoms that would indicate further investigation and/or cessation of hormonal contraception.

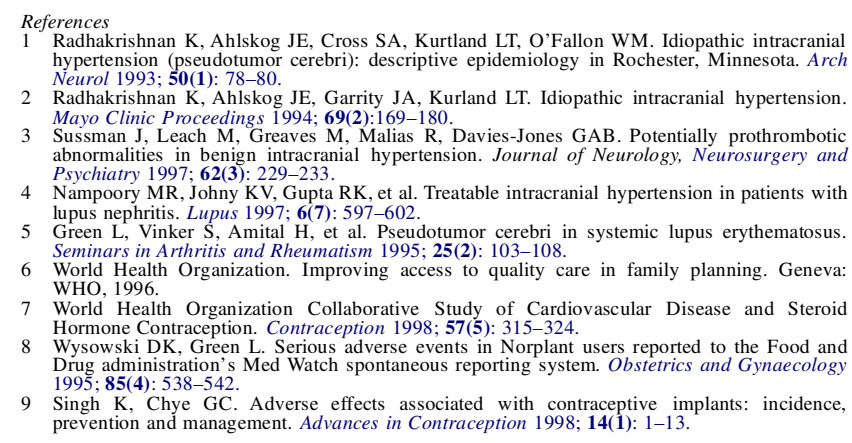

Radhakrishnan K, Ahlskog JE, Cross SA, Kurtland LT, O'Fallon WM. Idiopathic intracrania hypertension (pseudotumor

Radhakrishnan K, Ahlskog JE, Garrit Sussman J, Leach M, Greaves M, Malias R, Davies-Jones GAB. Potentially prothrombotic abnormalities in benign intracranial hypertension. Journal of Neurology, Neurosurgery and

lupus nephritis. Lupus 1997; 6(7): 597-602.

Seminars in Arthritis and Rheumatism 1995; 25(2): 103-108. WHO, 1996

and Steroi Hormone Contraception. Contraception 1998; 57(5): 315-324. Singh K. Chye GC. Adverse effects associated with contraceptive implants: incidence, prevention and management. Advances in Contraception 1998; 14(1): 1-13. 